\title{
Immagini religiose del mondo romano nella letteratura scolastica del Brasile contemporaneo
}

Religious Images of the Roman World in the Schoolbooks of Contemporary Brazil

Jorwan Costa Junior

\section{(2) OpenEdition}

1 Journals

\section{Edizione digitale}

URL: https://journals.openedition.org/mythos/3749

DOI: $10.4000 /$ mythos.3749

ISSN: 2037-7746

\section{Editore}

Salvatore Sciascia Editore

\section{Notizia bibliografica digitale}

Jorwan Costa Junior, «Immagini religiose del mondo romano nella letteratura scolastica del Brasile contemporaneo», Mythos [Online], 15 | 2021, online dal 15 décembre 2021, consultato il 15 décembre 2021. URL: http://journals.openedition.org/mythos/3749; DOI: https://doi.org/10.4000/mythos.3749

Questo documento è stato generato automaticamente il 15 décembre 2021.

Mythos 


\section{Immagini religiose del mondo romano nella letteratura scolastica del Brasile contemporaneo}

Religious Images of the Roman World in the Schoolbooks of Contemporary Brazil

Jorwan Costa Junior

\section{Introduzione}

1 Questo articolo si propone di analizzare le immagini di contenuto religioso inserite a corredo delle sezioni dedicate alla storia antica nei testi didattici oggi in uso nelle scuole brasiliane nel quadro dell'Ensino Médio (dai 15 ai 17 anni). Si tenterà di comprendere come queste immagini contribuiscano alla costruzione della conoscenza su Roma antica, inquadrino un orizzonte di discorso sulla classicità, e stabiliscano un dialogo con la realtà religiosa del Brasile contemporaneo. La documentazione che si discuterà è costituita dai manuali didattici approvati dal governo federale brasiliano nel ciclo triennale 2018-20201 , l'ultimo a essere giunto a compimento: tredici titoli in totale. Nell'affrontare il tema, occorrerà non perdere di vista i rapporti fra educazione pubblica e politiche governative, nei quali la produzione della letteratura scolastica ha un ruolo centrale. Questo quadro di riferimento è decisivo in un'indagine sulle modalità in cui la storia antica viene discussa attraverso i materiali didattici. Un'ipotesi di lavoro di questo studio è che i Reception Studies possano offrire importanti strumenti metodologici adeguati all'analisi, che sarà qui condotta attraverso due passaggi fondamentali. Attraverso un'indagine quantitativa, si tenterà di porre in risalto i temi e le omissioni ricorrenti che si possono riconoscere nell'associazione fra testo e immagini religiose proposta nei capitoli dedicati a Roma antica. In seguito, analizzeremo individualmente alcune illustrazioni, con l'intento di verificare l'ipotesi di lettura dalla quale prendiamo le mosse: nei manuali didattici brasiliani le immagini religiose riferite a Roma antica tendono a esaltare il cristianesimo e a sminuire il ruolo della religione romana. 
2 Si privilegia qui la dimensione iconografica dei libri scolastici, perché questo materiale ha negli ultimi anni subìto trasformazioni editoriali che ne hanno affinato la qualità grafica e hanno consolidato l'uso delle immagini come uno strumento pedagogico fondamentale nella costruzione della conoscenza. L'aspetto visivo è inoltre centrale in ogni tentativo di comprensione della religione romana, che era connessa all'identità civica dei cittadini, in un processo nel quale i rituali e le forme di rappresentazione del divino erano alla base della comunicazione fra gli attori coinvolti ${ }^{2}$. In uno scenario religioso privo di un'ortodossia o di sacre scritture, la corporeità degli dèi si affermava come uno degli elementi distintivi della loro identità, segnava le frontiere che li separavano dai mortali e si connetteva direttamente al potere stesso di Roma, rafforzando anzitutto il carattere divino dell'imperatore ${ }^{3}$.

\section{Insegnamento della Storia antica, Reception Studies e PNLD}

3 Le immagini religiose sono parte integrante dei manuali didattici di Storia adottati nel quadro dell'insegnamento medio, approvati dal Programa Nacional do Livro Didático (PNLD-2018). Si tratta di un'iniziativa mirata alla valutazione preventiva del materiale didattico in uso in tutte le scuole statali brasiliane, frequentate da più dell' $80 \%$ degli studenti che accedono all'insegnamento primario in Brasile: una platea, dunque, di migliaia di scuole e milioni di ragazzi ${ }^{4}$. Il PLND coordina la produzione della letteratura didattica e determina le scadenze alle quali le case editrici coinvolte nel programma governativo devono attenersi. Questo contesto normativo mette in risalto un tratto caratteristico dell'educazione scolastica brasiliana: la necessità dell'approvazione governativa per l'adozione dei testi didattici nelle scuole di Stato ${ }^{5}$. Questa caratteristica non è una prerogativa del sistema educativo brasiliano: anche in paesi molto diversi culturalmente e politicamente, come la Grecia, Cipro o Malta, le scuole, e in particolare quelle statali, utilizzano libri approvati da organi governativi ${ }^{6}$. Nonostante in Brasile la responsabilità principale per l'istruzione di base (dalla scuola per l'infanzia all'Ensino Médio) competa agli stati e ai municipi, gli enti locali sono obbligati a limitare i loro acquisti di materiali didattici a collane di libri di testo approvati ufficialmente dal PNLD. In linea teorica, questo non impedirebbe l'utilizzo di altri materiali didattici, o persino il rifiuto di servirsi di libri di testo approvati ufficialmente, sostituendoli con materiali prodotti a livello statale o municipale. Nella pratica, però, si riscontra un utilizzo pressoché esclusivo di materiali didattici approvati nel quadro del PNLD. Nel 2017 furono approvati per l'uso nell'insegnamento medio nel triennio successivo (2018-2020) soltanto tredici volumi che, in linea di principio, rispettavano il complesso dei processi politici e giuridici che regolano l'educazione di base in Brasile. Se ne possono riassumere $\mathrm{i}$ principi di fondo in alcuni punti qualificanti: ribadire l'importanza della lunga durata nello sviluppo storico; porre la costruzione della società in rapporto alle azioni politiche, economiche e sociali degli attori storici; contestualizzare le produzioni culturali; tutelare la diversità di genere, religione e orientamento sessuale; difendere la democrazia; promuovere la comprensione critica e analitica negli studenti; creare cittadini attivi nel mondo attuale ${ }^{7}$. Il PNLD orienta la produzione del materiale didattico: può dunque essere uno strumento decisivo nella difesa e nella promozione di un modello di società e di Stato fondato sul pluralismo politico e religioso ${ }^{8}$. 
4 L'insegnamento della Storia antica in Brasile propone uno studio della società grecoromana fondato su una letteratura specialistica spesso non più attuale ${ }^{9}$. Vi prevale una bibliografia prodotta per lo più fra gli anni Sessanta e Novanta del secolo scorso, che tende a porre al centro le fonti letterarie ${ }^{10}$. La tendenza di fondo è quella di riprodurre in maniera acritica la visione di un impero romano politicamente unito, senza dissensi o resistenze, socialmente omogeneo e a maggioranza cristiana. Da tempo e da più parti si rivendica dunque un insegnamento rinnovato della Storia antica, che possa esplicarsi anche nella letteratura scolastica. Fra l'ambito della ricerca universitaria e quello dell'insegnamento scolastico, peraltro, non vi è uno scambio fruttuoso. L'approccio alla documentazione è rivelatore in questo senso. Nella pratica didattica si osserva l'assenza di riferimento a fonti letterarie e materiali che permettano allo studente di riflettere sull'antichità a partire da frammenti di documentazione primaria. Quando sono presentate, le fonti antiche hanno un carattere meramente illustrativo, e non alimentano il dibattito e la discussione critica fra gli studenti. Condividono inoltre lo spazio nelle pagine del libro di testo con testi e immagini non-classici che interpretano, rappresentano e rileggono aspetti della civiltà classica ${ }^{11}$.

Questo stato di cose suscita a sua volta una domanda fondamentale: se fra gli autori dei libri didattici riconosciuti nel PNLD-2018 vi siano autori con una competenza disciplinare specifica in Storia antica. Per rispondere a questo interrogativo si può fare ricorso a due tipi di materiali: i libri di testo e la piattaforma Lattes ${ }^{12}$. Emerge con chiarezza che i manuali di storia del PNLD non sono necessariamente scritti da storici: su un totale di ventotto autori, quattro hanno una formazione universitaria di base in Storia, nell'ambito di percorsi di studio in Biblioteconomia, Scienze Sociali, Pedagogia e Diritto. Tre autori hanno una formazione universitaria avanzata in Storia, ma non viene specificato in quale disciplina abbiano compiuto i loro studi di base. Ventuno autori - la maggioranza - hanno una qualificazione universitaria in Storia. Diciotto hanno titoli universitari avanzati, e nove di essi un dottorato. Nessuno ha un dottorato in Storia antica.

6 Le immagini a tema religioso incluse nei capitoli dedicati a Roma e i testi che le accompagnano trasmettono discorsi testuali e visivi intorno ai classici forgiati in contesti storico-culturali differenti, che propongono tesi talvolta contrastanti rispetto a quelle proposte dalla ricerca recente o attuale. Analizzarli a partire dall'angolazione teorica dei Reception Studies permette di identificare in quali contesti politici, sociali e religiosi si collochino tali reinterpretazioni del classico, svelando la logica con cui la nozione di "classico" è costantemente prodotta dai contesti culturali in cui viene indagato. È dunque necessario intendere anche i limiti interpretativi imposti dalle circostanze in cui il classico viene (ri)prodotto. L'indagine proposta qui si richiama dunque alle correnti storiografiche che, negli ultimi decenni, hanno sostenuto l'idea secondo cui lo studio dei discorsi riguardo al Classico e i classici è parte integrante degli studi di antichistica ${ }^{13}$.

In linea teorica, il manuale didattico dovrebbe contribuire alla costruzione di una cittadinanza critica e promuovere la difesa della democrazia e del pluralismo, specialmente in campo religioso. Nel campo dell'insegnamento della Storia, sarebbe inoltre necessario porre l'accento sugli elementi che illustrano come la costruzione della conoscenza storica sia l'effetto di un processo. Questi princìpi di fondo dovrebbero poi esplicarsi in un insegnamento dell'antichità, specialmente di quella romana, che ne esalti gli aspetti improntati al pluralismo, in particolare nell'ambito 
religioso. Raggiungere tali obiettivi fondandosi su libri scritti da non-classicisti, senza entrare in dialogo con le fonti antiche, è un'impresa ardua. L'assenza di una formazione storica degli autori complica seriamente l'uso delle fonti antiche come documenti storici, che vengono trascurate nella letteratura didattica in due modi: omettendo $i$ riferimenti ad esse o nei loro usi come illustrazioni.

Tornando al tema specifico di questo contributo, l'uso delle immagini religiose del mondo romano nella letteratura scolastica brasiliana pone alcune grandi questioni: qual è il contesto di produzione delle immagini scelte dagli autori? Come sono distribuite all'interno del libro? Quali sono i temi ricorrenti e le omissioni? Vi è un qualche grado di varietà nelle sue rappresentazioni? È possibile percepire il peso del contesto religioso brasiliano nella selezione delle immagini usate nei libri scolastici? Che dialogo si instaura fra le immagini rappresentate e le esperienze religiose del Brasile moderno?

\section{Le immagini religiose: temi ricorrenti e omissioni}

Una breve analisi quantitativa dei dati relativi alla totalità delle immagini religiose incluse nella letteratura didattica in Brasile, la loro cronologia, gli esseri divini che rappresentano e le religioni alle quali fanno riferimento permette uno sguardo panoramico su questi libri di scuola e la discussione di alcune immagini specifiche. Per gli intenti di questo lavoro si sono considerate le "immagini religiose" in senso lato, includendo dunque ogni illustrazione che in un libro di scuola faccia riferimento a un edificio o a un luogo di culto, rappresenti un essere divino o contenga un simbolo religioso.

10 Nei tredici volumi inclusi nel PNLD-2018, attualmente utilizzati nel quadro dell'Ensino Médio brasiliano ${ }^{14}$, abbiamo identificato 33 immagini religiose su vari supporti: affreschi, pitture, rilievi, statue ed edifici. Non esiste un equilibrio nelle occorrenze delle immagini, poiché, anche se un libro non presenta alcun tipo di immagine religiosa, altri ne includono addirittura cinque. In questi volumi si nota, peraltro, la codificazione di due "religioni" su cui si polarizza il dibattito: il "politeismo romano", con diciotto immagini, e il "cristianesimo", con quindici ${ }^{15}$. Fra le immagini non cristiane, solo due non rappresentano delle divinità ${ }^{16}$ : la prima è una fotografia del Pantheon, priva di datazione ${ }^{17}$; l'altra rappresenta la statua di una sacerdotessa pompeiana di età augustea, Eumachia ${ }^{18}$. Fra le divinità romane, Minerva è rappresentata in due contesti: in un larario di Napoli del primo secolo d.C. e in una statua rinvenuta a Roma dello stesso periodo ${ }^{19}$. In un larario, anch'esso di primo secolo, proveniente dalla Casa dei Vetti, sono rappresentati due Lari ${ }^{20}$. Marte appare nell'Ara di Domizio Enobarbo, della fine del secondo secolo a.C., che rappresenta un censimento romano e un rito che lo accompagna ${ }^{21}$. Venere è ritratta in due occasioni: con la celebre statua della Venere di Milo, risalente al secondo secolo a.C., e con la Venere di Fréjus, la cui datazione non è indicata, nonostante sia specificato che si tratta di una copia romana di un'opera prodotta nel quinto secolo a.C. ${ }^{22}$. Cupido è rappresentato in sei casi: accanto a Psiche, in una statua del diciassettesimo secolo, e in cinque immagini della statua di Augusto di Prima Porta ${ }^{23}$. A Iustitia fa riferimento la riproduzione di una statua, oggi a Francoforte, la cui cronologia non viene precisata ${ }^{24}$. Athena Promachos è ritratta in una statua, presentata come una replica romana, del primo secolo ${ }^{25}$. Un 
mosaico del terzo secolo d.C. rappresenta Virgilio fra Clio e Melpomene ${ }^{26}$. Infine, Nettuno e Anfitrite appaiono in un mosaico del quarto secolo ${ }^{27}$.

11 Sul versante cristiano, si osservano sei immagini che non rappresentano Gesù l'affresco del Beato Angelico, L'Annunciazione (1440-1445), con Maria e l'Angelo Gabriele ${ }^{28}$; il quadro A primeira missa no Brasil, di Victor Meirelles, del $1860^{29}$; il dipinto ottocentesco Triomphe de la Foi, opera di Eugène Thirion ${ }^{30}$; le Catacombe di San Gennaro $^{31}$ e di Priscilla ${ }^{32}$ sono presentate come luoghi di culto cristiani, senza specificarne data e localizzazione; e, infine, la sopraccitata foto del Pantheon, indicato anche come un tempio cristiano a partire dal sesto secolo ${ }^{33}$. Immagini di Gesù appaiono in nove occasioni. Un Gesù risorto appare in un dettaglio del dipinto Madonna con Bambino e due Santi di Francesco di Giorgio Martini, del $1495^{34}$. Un Cristo ritratto in età adulta appare in due occasioni nell'Affresco del Buon Pastore, del terzo secolo ${ }^{35}$. In due casi Gesù viene rappresentato attraverso attori cinematografici che lo interpretarono, rispettivamente in The Color of the Cross, del 2006, e in The King of the Kings, del $1961^{36}$. Altri due libri lo mostrano in due mosaici: uno del quinto secolo, nella Cappella di Sant'Andrea a Ravenna ${ }^{37}$, in cui Cristo appare in vesti da legionario romano, e un altro del quarto secolo, proveniente dalla Basilica di Santa Pudenziana a Roma, nel quale appare seduto in trono ${ }^{38}$. In un dipinto del quarto secolo, nelle Catacombe dei Santi Pietro e Marcello, Gesù è rappresentato mentre compie un miracolo, noto come la Guarigione dell'Emorroissa ${ }^{39}$. Vi è infine l'immagine di un sarcofago risalente al quarto secolo, nel quale sono rappresentate scene della Passione di Cristo ${ }^{40}$.

Il primo aspetto che emerge da questa rapida rassegna indica una falsa equivalenza fra il numero totale di immagini riservate a ciascuna religione. I libri didattici presentano la storia di una città attraverso 1100 anni, dalla sua fondazione, nel 753 a.C., sino alla caduta dell'impero romano d'Occidente, nel 476 d.C. ${ }^{41}$. Nel caso del cristianesimo, si osserva uno spazio temporale di poco più di 400 anni nel contesto dell'insegnamento scolastico di Roma antica. In tutto l'insieme dei libri didattici quindici immagini, all'incirca, sono state dunque utilizzate per rappresentare il cristianesimo. Se si osservano i dati di produzione nel grafico della figura 1, emergono poi altri spunti di analisi.

Fig. 1: Grafico dei dati di produzione di immagini cristiane e non cristiane

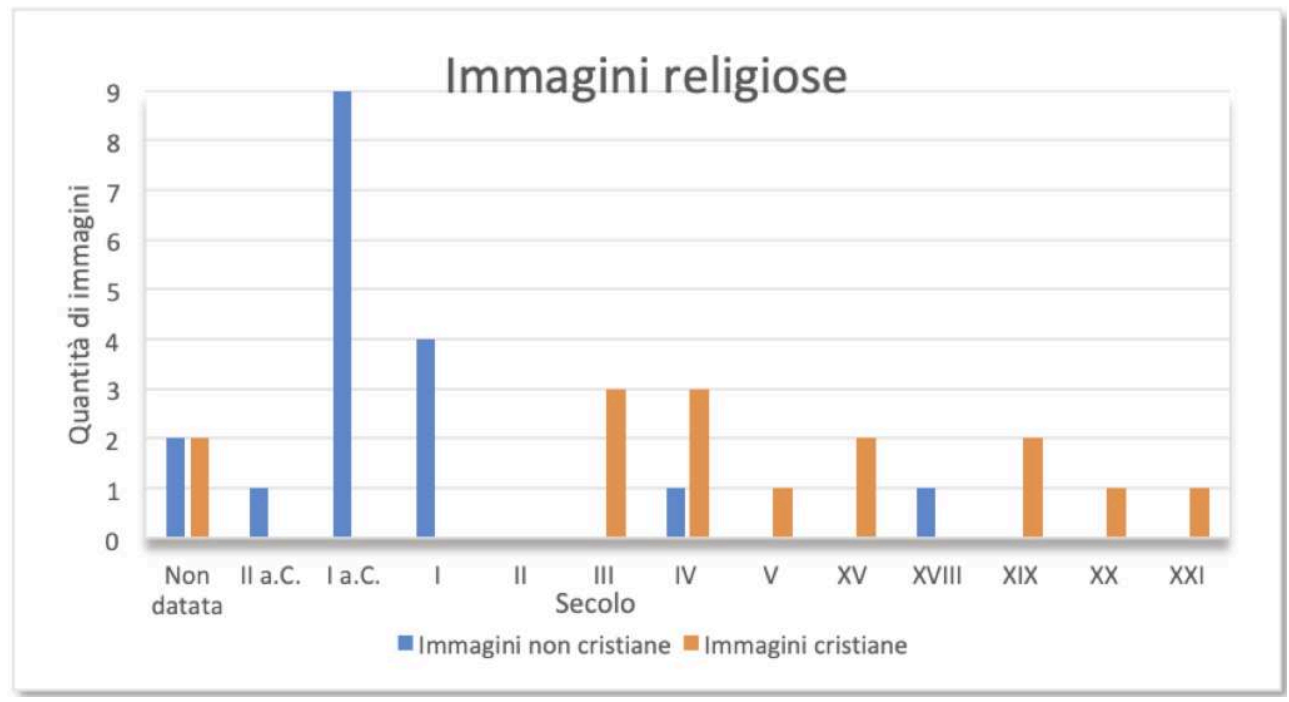


13 Riguardo alla religione romana, il grafico mostra un'assenza quasi totale di immagini prodotte prima del primo secolo a.C. Poiché si tratta di un materiale rivolto all'insegnamento scolastico, questa scarsità può generare la falsa impressione che vi siano scarse testimonianze iconografiche per questo periodo o, persino, l'idea che semplicemente non vi fosse una religione prima di questa data. Anche quando sono rappresentate, le immagini non permettono di costruire una conoscenza solida sul tema. In quanto aggregato di simboli, pratiche e tradizioni, la religione romana aveva i propri riti, sacrifici e feste: elementi fondativi del paesaggio religioso, oltre che temi predominanti nell'arte romana. Nel caso dell'Ara di Domizio Enobarbo, l'immagine viene esposta senza alcuna contestualizzazione, impedendo agli allievi di stabilire alcun tipo di rapporto fra divinità e rituali ${ }^{42}$. Questa mancata caratterizzazione della religione romana trova un'eco nella presentazione del Pantheon, che viene privato del suo carattere religioso romano, poiché la didascalia lo presenta come un tempio cristiano a partire dal sesto secolo d.C., senza spiegarne la funzione prima della sua cristianizzazione, o persino le date della sua costruzione e ricostruzione. È infine significativo che non vi siano immagini di sorta della Triade Capitolina e che soltanto la dea Minerva sia esplicitamente presentata al lettore. Mentre Giove e Giunone sono accuratamente evitati, Gesù viene ricordato ripetutamente. Gesù è, come è logico attendersi, la presenza centrale nello sviluppo storico del cristianesimo e la frequente riproduzione della sua immagine è un fatto ovvio. La religione romana si fonda su principi di rappresentazione del divino ben diversi; un'equivalenza fra le divinità romane e Cristo sarebbe fuorviante. D'altra parte, sarebbe auspicabile vedere riprodotta in un libro di testo un'immagine della Triade Capitolina accanto a quella di Gesù, sulla stessa scala.

14 La dinamica discussa sin qui denota dunque una precisa strategia iconografica, che riduce al silenzio la religione romana, aggravata dal fatto che molte delle divinità rappresentate non sono neppure sono citate nei testi dei capitoli, ma appaiono senza ulteriore contestualizzazione nell'apparato illustrativo. Una rassegna delle espressioni riferite alla religione porta a individuare cinque termini ricorrenti: "cristianismo", "religião cristã"; "religião romana"; "politeísmo romano" e "religião tradicional romana". Il termine "cristianismo" appare in 63 occasioni e "religião cristã" in quattro. L'espressione "religião romana" è utilizzata in due sole occasioni; "politeísmo romano" e "religião tradicional romana" una volta ciascuna. È significativo che l'espressione "religione romana" sia utilizzata con grande riluttanza; né viene data una ragione per questa chiara scelta terminologica. Si tratta dunque di un processo che riduce la religione romana nei libri scolastici a un ruolo minimo, a fronte dell'ipertrofia delle discussioni incentrate sul cristianesimo.

15 I libri scolastici privilegiano le tematiche riferite all'impero romano e alla sua espansione nel Mediterraneo; anche in questo contesto, non vi è alcuna rappresentazione di divinità che non siano (greco-)romane o cristiane. Per quanto riguarda le immagini religiose non cristiane, vi è un chiaro predominio di alcuni temi: in particolare, l'età augustea e la pax Romana. Le immagini religiose riflettono questa prevalenza con cinque riproduzioni dell'Augusto di Prima Porta, dove il princeps appare accanto a Cupido, che emerge come un'immagine allegorica di fronte al potere imperiale. La focalizzazione sulla figura politica di Augusto ignora che il potere di Augusto fu giustificato, in larga misura, con l'ascendenza divina della gens Julia e con un preteso appoggio divino, manifestato nella figura di Cupido, figlio di Venere, 
progenitrice della gens Julia. Questo complica di molto la comprensione del rapporto fra politica e religione nel mondo romana.

Il ricorso a una sola immagine religiosa non cristiana prodotta nei secoli III-IV d.C. è in netto contrasto con le sei immagini cristiane identificabili per lo stesso periodo, e si salda a un messaggio politico, che vede in questi secoli un periodo di crisi dell'impero romano, intimamente legato all'ascesa del cristianesimo. L'esibizione di altre immagini religiose non cristiane indebolirebbe la narrazione teleologica di un'avanzata del cristianesimo. Tale approccio sarebbe inoltre coerente con una tradizionale divisione dell'insegnamento della storia di Roma ripartita fra fondazione, età monarchica, repubblica, Impero, disgregazione e caduta dell'Impero. Il grafico mostra un maggior numero di immagini cristiane moderne, prodotte fra il XV e il XXI secolo. Nessuna di esse, peraltro, è collocata, pensata o discussa alla luce dei suoi contesti di produzione: la sua funzione è meramente illustrativa. Si tratta prevalentemente di immagini isolate, che non svolgono la funzione didattica di fonti storiche, e dunque non contribuiscono alla costruzione di una riflessione critica sull'ascesa e il consolidamento del cristianesimo nell'ambito della società romana. Le immagini mirano dunque a rafforzare la concezione di un valore atemporale del cristianesimo, il suo ruolo egemonico nell'Occidente e la sua presunta funzione determinante nella disgregazione dell'impero romano.

17 La dinamica dei temi e delle omissioni ricorrenti discussa sin qui è stata studiata anche da Simon Goldhill, secondo cui il consolidarsi del cristianesimo nel mondo moderno è stato accompagnato da un processo di oblio e di oscuramento della poesia greca, a favore di quella romana, meglio adattabile a un'eteronormatività cristiana ${ }^{43}$. I libri didattici brasiliani replicano questo modello, promuovendo l'oblio o l'oscuramento della religione romana di fronte a un'ipertrofia del cristianesimo. In questo contesto, sarebbe possibile comprendere la vita religiosa di un popolo senza rappresentarne gli dèi e gli elementi religiosi più rilevanti? Può esservi una valorizzazione della varietà religiosa in cui agli allievi non viene proposta la rappresentazione di contesti religiosi diversi dal loro? ̇̀ possibile stabilire un insegnamento della Storia antica effettivamente critico, svalutando il peso politico e sociale della religione nella Roma antica?

Le risposte negative a tali domande mettono in risalto come le immagini riunite nei libri di testo attualmente in uso in Brasile non siano in linea con gli sviluppi più recenti negli studi classici nel XXI secolo e, al tempo stesso, non rafforzino la varietà religiosa incoraggiata dagli strumenti normativi che regolano l'istruzione in Brasile e la produzione di libri didattici. L'assenza di antichisti, o comunque di autori con una formazione classica, nell'elaborazione dei libri di testo e la scarsa diffusione degli studi classici nelle università brasiliane possono spiegare in parte l'uso acritico delle immagini religiose romane e la continua presenza dell'immagine di Gesù, che rafforzano l'idea di una tradizione cristiana brasiliana e offrono un insegnamento scolastico di Roma antica come "culla del cristianesimo" ${ }^{44}$. Una rapida analisi qualitativa delle immagini potrà portare ulteriori conferme a questa lettura.

\section{Le immagini nei libri}

19 Poiché ciascuna di queste immagini va analizzata attraverso i vari elementi che la compongono, e poiché è necessario non perderne di vista la collocazione in un libro di 
scuola, nelle pagine che seguono si analizzeranno le didascalie che accompagnano le immagini, la posizione delle immagini nella pagina, la logica della collocazione delle immagini in un capitolo e le interazioni fra immagini riprodotte su una medesima pagina $^{45}$. Per ragioni di sintesi, si sono selezionati quattro esempi tratti dalle ventitré immagini elencate prima.

Il primo riguarda l'uso dell'immagine della statua di Athena Promachos (Fig. 1), nel libro di testo História Passado e Presente. La statua si colloca accanto al sottotitolo 4 Cultura Greco-romana, condividendo lo spazio con un testo alla sua destra e con una serie di attività didattiche nella parte inferiore. In questo libro di testo la sezione precedente a quella in cui Atena è rappresentata è dedicata all'età repubblicana, e si concentra su sviluppi politici e militari di grande importanza, quale l'espansione mediterranea e le rivendicazioni della plebe per una maggiore inclusione politica. La sezione immediatamente successiva all'immagine di Atena, intitolata "Declino della Repubblica", è anch'essa centrale negli sviluppi politici e militari fra il secondo e il primo secolo a.C. Non vi sono nessi logici o argomentativi che permettano di identificare il legame della dea $\mathrm{o}$, più in generale, della religione greca o romana con la quotidianità della vita politica di Roma. La didascalia che accompagna l'immagine è inoltre assai discutibile. L'espressione "religione romana" non appare nella pagina, e allude a una pretesa idea secondo cui la si potrebbe ridurre a una copia della religione greca, poiché la didascalia sottolinea come la statua riproduca un originale greco; il testo stabilisce un'equivalenza semplicistica fra i nomi degli dèi greci e di quelli romani, messa in risalto dall'unica menzione della religione romana nel testo che accompagna l'immagine: "Na religião, os romanos passaram a venerar os deuses gregos, embora os romanos tenham imposto nomes latinos para eles [...]" ${ }^{\text {" }}$. La dea greca è rappresentata in un capitolo dedicato al mondo romano, inserito in una "Cultura greco-romana", come indica il titolo della sezione. In questo modo, gli stretti e complessi rapporti culturali fra greci e romani sono ridotte a un mondo nel quale entrambe le culture condividevano una visione del cosmo. La divinità greca messa in risalto dall'immagine non aiuta a comprendere la religione romana nel suo contesto storico. Non vi è alcun cenno alla statua nel testo: alla sua funzione religiosa, al suo significato, o anche all'azione che sembra esservi rappresentata. Allo stesso modo, le sezioni che seguono non la citano neppure, e si riferiscono appena ad aspetti politici o militari della storia romana. La riproduzione della statua di Athena Promachos è dunque esposta come un'illustrazione svuotata di qualunque significato storico in grado di contribuire alla costruzione della conoscenza sulla religione romana. 
Fig. 2 Statua di Athena Promachos riprodotta nel libro di testo História Passado e Presente

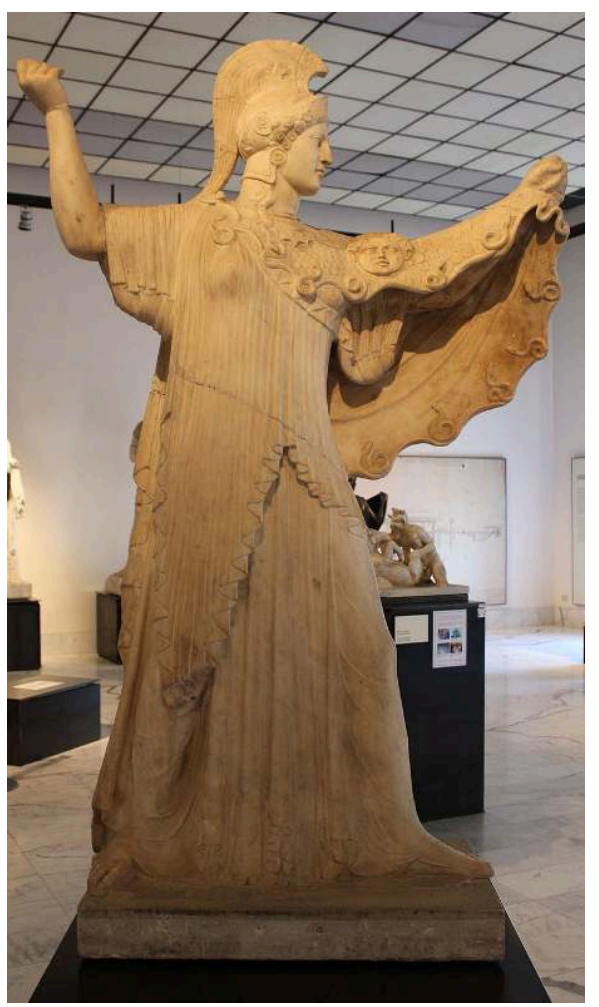

AzeVedo, Seriacopi 2016, 140; licenza CC-BY SA 3.047

21 Un'altra divinità romana alla quale si fa ampio riferimento è Cupido. Il libro di testo Cenas de História include nella stessa area tematica una statua di Amore e Psiche di Antonio Canova accanto alla copertina di un'edizione moderna dell'Ars amatoria di Ovidio ${ }^{48}$. L'autore definisce la nozione di amore come una connessione diretta fra il mondo di oggi e quello romano. La centralità degli esseri divini si contrappone al testo in cui sono citati, che si riproduce qui di seguito:

$\mathrm{Na}$ mitologia romana, que teve origem nos mitos gregos, Cupido, o deus do Amor, vai ao Inferno para resgatar Psiquê, por quem ele se apaixonou. A obra do artista Antonio Canova (1757-1822), nascido na cidade de Possagno, na atual Itália, imortalizou o casal no momento em que cupido toma Psiquê nos braços e é considerada a representação ideal do amor romântico: quem ama é capaz de tudo (até de descer aos infernos) para dar felicidade ao ser amado. Porém, apesar de ter surgido de um mito da época da Roma Antiga, essa ideia de amor romântico é muito recente e bem diferente do modo como Ovídio, por exemplo, descreve as relações entre os casais naquela sociedade ${ }^{49}$.

Il testo associa Amore e Psiche all'idea di amore romantico, ponendola in rapporto a Ovidio: un'operazione altamente discutibile. La statua, peraltro, è utilizzata per rappresentare un'idealizzazione moderna e borghese dell'amore, che difficilmente si può mettere in dialogo con l'Antichità. Amore e Psiche, e Ovidio stesso, sono dunque usati per definire concezioni postclassiche dell'amore. Non vi è alcun nesso fra questo inquadramento iniziale del mondo romano con i contenuti discussi nel resto del capitolo, che si concentra su problemi di carattere politico-sociale. Cupido appare poi in altri cinque volumi didattici, sempre ai piedi di Augusto, ritratto nella celebre statua di Prima Porta ${ }^{50}$. 
23 Va poi messo in risalto l'uso, nel libro di testo Cenas de História, del dipinto A Primeira Missa no Brasil (Fig. 2) di Victor Meirelles (1861), che illustra l'apertura di un capitolo dedicato alla "caduta" dell'impero romano in Occidente ${ }^{51}$. Esso fa riferimento alla prima messa celebrata in Brasile, nel 1500, nella città di Santa Cruz de Cabrália. La scelta implica la volontà di evidenziare la centralità del cristianesimo nel processo di colonizzazione portoghese in Brasile: il centro dell'immagine, dove è posta la croce, è più illuminato dei margini del quadro. Gli indigeni manifestano un misto di incredulità, paura e rispetto nei confronti della cerimonia, e gli altri personaggi sono in una posizione deferente. Il testo che l'accompagna propone un'affermazione più che discutibile:

Em outras palavras, saíam de cena as sociedades organizadas em torno de cidades, como Roma e Alexandria, e entravam em cena povos regidos pela Igreja Cristã, por valores ligados ao cristianismo. Inauguravam-se, em resumo, novas formas de vida e sociedade ${ }^{52}$.

L'immagine è accompagnata da un testo che sottolinea il ruolo del cristianesimo come una componente cruciale nell'identità brasiliana e trae origine nell'ambito dell'impero romano. Testo e immagine alludono dunque, entrambi, a una trasmissione ininterrotta della tradizione cristiana, che trae origine dal passato classico, ed è in stretto rapporto con la vicenda del Brasile di oggi ${ }^{53}$. Il Brasile del secolo XIX, l'epoca in cui fu prodotto il quadro, assistette a una politica di costruzione dell'identità nazionale incentrata sul cristianesimo, sulla sottomissione degli indigeni ad esso e sull'avvento del regime imperiale come fattore di coesione politica nel paese. Nell'aprire un capitolo dedicato allo studio di Roma antica con quell'immagine, il libro didattico del secolo XXI valorizza il cristianesimo di fronte al crollo dell'impero romano, e vi conferisce il ruolo di elemento unificatore di società antiche, moderne, americane o europee. Si afferma dunque una duplice vittoria del cristianesimo: su Roma e sugli indigeni brasiliani. 


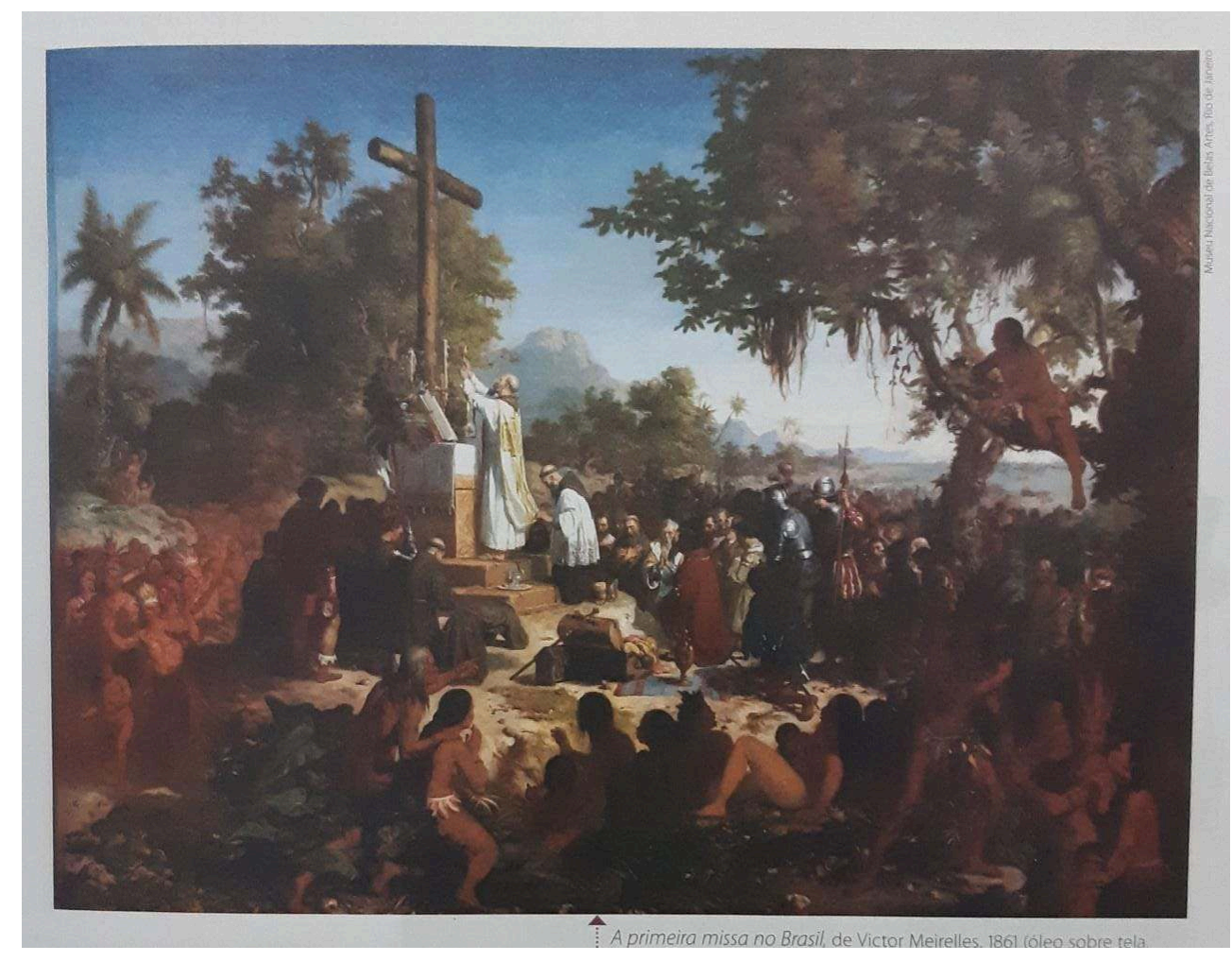

Disponibile a: https://commons.wikimedia.org/wiki/

File:Pintura_sobre_a_primeira_missa_no_Brasil.jpg. Ultimo accesso: 12.11.20

In un altro libro, História Global54, l'associazione fra il cristianesimo e la disgregazione dell'impero romano si ripete nella presentazione del dipinto Triomphe de la Foi: Martyrs Chrétiens au Temps de Néron en 65, opera non datata del pittore francese Eugène Thirion $(1839-1910)^{55}$. Fra gli sconfitti e i feriti, una figura maschile, uno dei martiri a cui allude il titolo, si colloca al centro dell'arena e guarda al cielo, accanto alla croce, che è collocata su un rudimentale altare, e la distingue rispetto a tutti gli altri elementi della scena. L'opera si colloca in una narrazione bellica: passato il periodo "pagano" termine utilizzato due volte nel testo che precede l'immagine - i cristiani escono vittoriosi dalle battaglie per la vita e per la fede (Fig. 3). Questa immagine fa da cornice a una discussione degli aspetti religiosi a Roma, che dimostra di nuovo l'ipertrofia del cristianesimo. La sezione in cui si trova l'illustrazione si intitola "Religião: do politeísmo ao cristianismo". Vanno fatte due osservazioni al riguardo. La prima risiede nella mancata utilizzazione del termine "religione romana", sostituito da "politeísmo". Oltre a questo, si crea un'evoluzione inesorabile della società romana in direzione del cristianesimo, semplificando il processo nella sua interezza. Il contenuto relativo a questo aspetto religioso è suddiviso in quattro sezioni: tre sono dedicate al cristianesimo e soltanto una alla religione romana. L'immagine si colloca fra due titoli: Perseguição aos cristãos e Conquista da liberdade religiosa. Parole e immagini sottolineano il carattere bellico della vittoria del cristianesimo:

A maneira heroica com que muitos cristãos resistiam aos sofrimentos, nos martírios públicos, começou a ser interpretado como algo possível apenas devido a uma força milagrosa, vinda de Deus ${ }^{56}$. 
come, in un passo immediatamente successivo, la religione romana venga acriticamente associata al paganesimo: "Cerca de uma década depois, os cultos pagãos foram proibidos, e o cristianismo tornou-se, efetivamente, a religião oficial de Roma [...]"57. Il libro fa dunque suoi, e tenta di imporre, il termine elogiativo "eroe" per i martiri e lo spregiativo "pagano" per la religione romana.

Fig. 4. Triomphe de la Foi: Martyrs Chrétiens au Temps de Néron, di Eugène Thirion (1839-1910), non datato

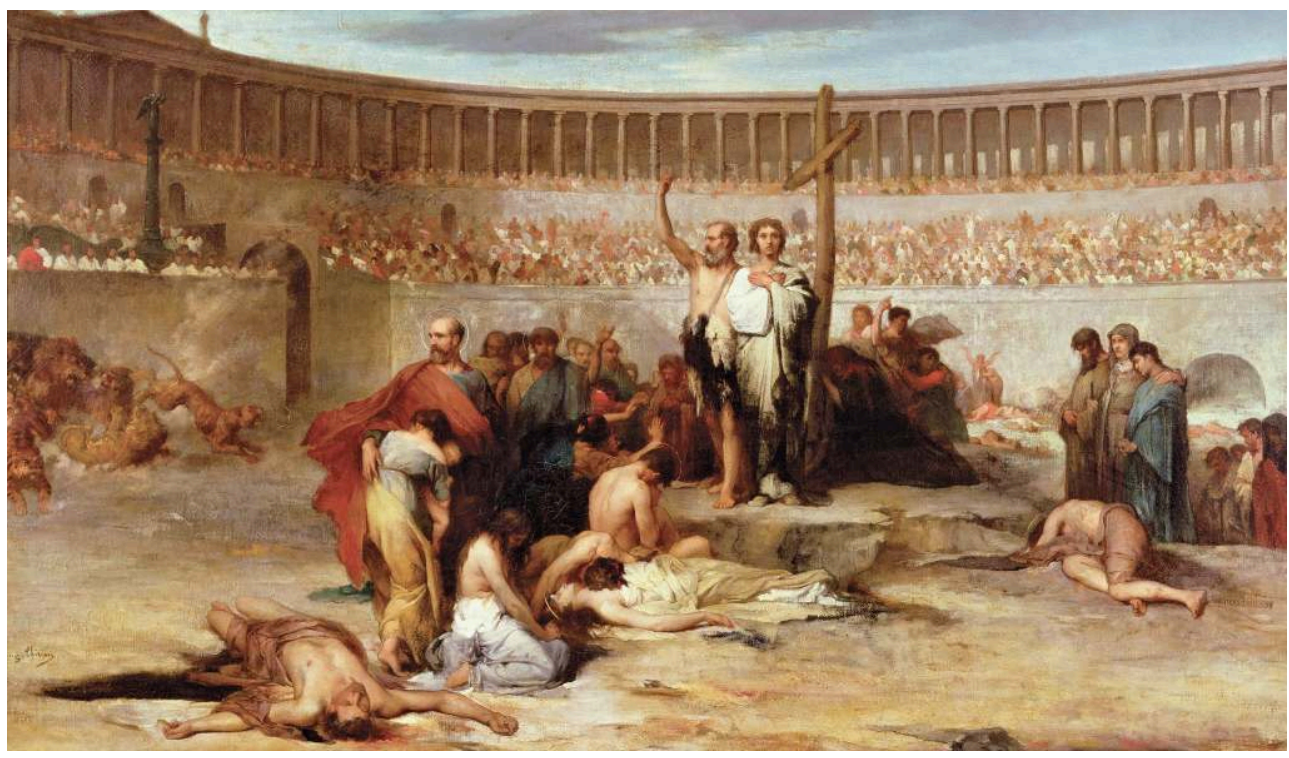

Disponibile a: https://fr.wikipedia.org/wiki/Eug\%C3\%A8ne_Thirion\#/media/ Fichier:Triumph_of_faith_by_Eugene_Thirion.jpg Ultimo accesso: 12.07.21

Dal quinto e ultimo esempio, tratto dal libro Olhares de História, emerge di nuovo l'intreccio del cristianesimo con l'impero e la logica bellica, illustrata da un mosaico del quinto secolo d.C., nel quale Gesù Cristo, in veste di legionario, assicura la croce sulle braccia, in modo che l'oggetto assomigli più a un'arma che a un simbolo religioso (Fig. 4). Cristo calpesta un leone con il piede destro e un serpente con il piede sinistro. Nella sua mano sinistra giace un libro con una legenda latina: "Io sono il cammino, la verità e la vita" ${ }^{58}$.

Nel libro scolastico, l'immagine è esposta con una didascalia che associa la disgregazione dell'impero romano all'espansione del cristianesimo: “A ascensão do cristianismo correspondeu à desagregação do Império Romano. Cristo é representado nesse mosaico do século $\mathrm{V}$ como un legionário que derrota o mal, simbolizado por un leão e serpente". D'altra parte, una frase nella stessa pagina dichiara che il cristianesimo era "contrário à violência", entrando in apparente contraddizione con quanto presentato dall'immagine e dal testo ${ }^{59}$. Si rafforzano così il carattere bellico e l'inevitabilità del cristianesimo, ponendo domande di fondo: che cos'è il male? Che cosa è l'impero? E che cos'è la religione romana? Unendo testi e immagini, un lettore potrebbe dedurre che Gesù non sia responsabile soltanto della caduta dell'impero, ma anche di quella del "politeismo". La politica e la religione romane potrebbero persino essere lette come la rappresentazione del "male" citato nella didascalia. 
Fig. 4. Mosaico di Cristo che calpesta le bestie, Cappella Arcivescovile, Ravenna, secolo VI

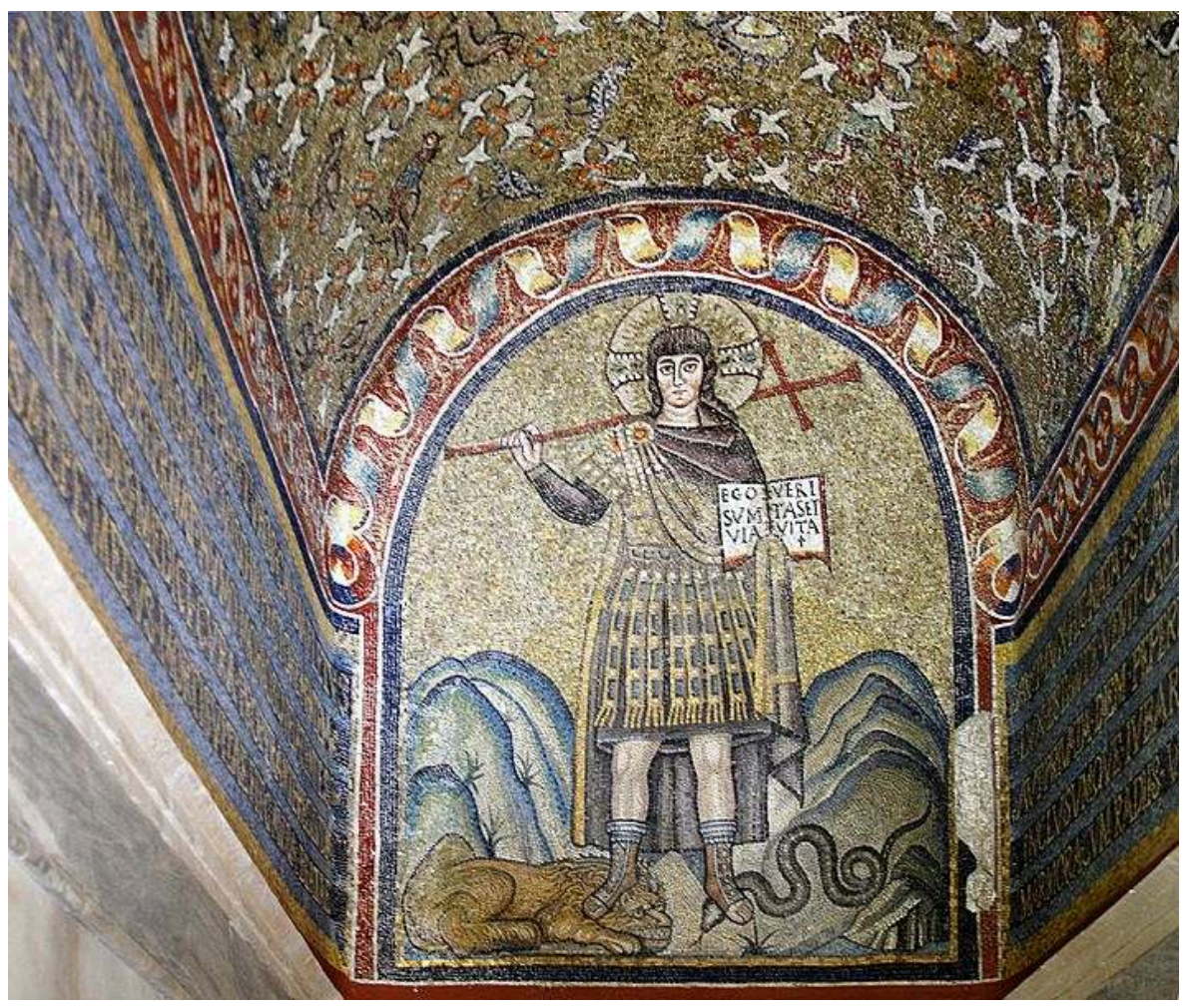

Disponibile a: https://commons.wikimedia.org/wiki/File:Cappella_arcivescovile_Ravenna_7.JPG, ultimo accesso 15.07.21

Gli usi di queste immagini permettono di identificare un modulo di ricezione del cristianesimo e della religione romana nei libri di testo in Brasile. I temi iconografici ricorrenti pongono in evidenza la costruzione di un panorama religioso che rafforza i legami identitari che il Brasile condivide con il cristianesimo sin dai tempi della colonizzazione portoghese. In una retorica di violenza, di omissione sistematica delle altre religioni e di rafforzamento dell'egemonia cristiana, le immagini analizzate qui non contribuiscono alla promozione della diversità religiosa, come pure richiedono gli strumenti normativi sui quali si fonda l'insegnamento della storia. ${ }^{60}$ Per quanto la verità storica delle lotte dei cristiani per la sopravvivenza delle loro pratiche religiose sia debitamente riconosciuta, i libri di testo mettono in risalto il trionfo del cristianesimo su un nemico religioso invisibile. La religione romana non è efficacemente spiegata al lettore e viene presentata in modo tendenzioso, come ha dimostrato l'analisi della Figura 4. Resta da comprendere il contesto della ricezione di questi discorsi, al fine di interpretare il compito che svolgono nella costruzione della conoscenza sull'antichità classica. In che modo il ricorso a queste immagini riflette, mette in dubbio o avvalora la realtà religiosa brasiliana del secolo XXI?

\section{Il contesto della ricezione}

Il giovane brasiliano che frequenta lo Ensino Médio è inserito in una società eminentemente cristiana sin dai tempi della colonizzazione portoghese. Questa tradizione cristiana si è trasformata negli ultimi decenni, con il progredire delle confessioni neopentecostali, che oggi rappresentano oltre il 30\% della popolazione 
brasiliana ${ }^{61}$. Il ruolo del cristianesimo nel passato e nel presente del Brasile è ormai solo una parte del contesto. Religioni di derivazione africana si sono diffuse in diverse regioni del paese, e hanno a lungo subito le conseguenze di una profonda intolleranza. Se nel passato vi è stata un'intensa azione della Chiesa Cattolica per sopprimere le manifestazioni religiose dei popoli africani schiavizzati in Brasile, in tempi più recenti anche alcuni gruppi neopentecostali sono stati responsabili di attacchi a religioni di matrice africana, come nel caso dei recenti attacchi a luoghi di culto candomblé a Rio de Janeiro e a Bahia ${ }^{62}$. Non a caso, queste sono le due regioni che hanno accolto il maggior numero di africani schiavizzati nel corso della storia del Paese.

31 A proposito di immagini religiose, va ricordato il Carnevale del 2017, nel quale la scuola di samba Estação Primeira de Mangueira, su pressione dell'Arcidiocesi di Rio de Janeiro, non presentò al Desfile das Campeãs (la prestigiosa «Sfilata delle Campionesse») una scultura in cui Gesù Cristo e Oxalá, divinità della Umbanda (una religione sincretistica afro-brasiliana), erano ritratti fianco a fianco ${ }^{63}$. L'educazione pubblica non è lontana da queste controversie. Al Congresso è stato depositato un progetto di legge che tenta di codificare l'obbligatorietà dell'insegnamento religioso, giustificandolo con l'intento di fondarlo sulla "religiosità brasiliana" ${ }^{64}$, termine fondamentalmente incongruo rispetto alla realtà religiosa del Paese, per quanto in essa vi sia indubbiamente un predominio cristiano. Questa realtà religiosa, nella quale gli allievi brasiliani sono inseriti, influenza direttamente le modalità con cui essi si misurano con il materiale didattico. Interessa qui osservare, anzitutto, il rapporto che si instaura fra i contesti di produzione del libro didattico in Brasile e quelli della sua ricezione.

Il cristianesimo ha nel territorio brasiliano una presenza capillare. Nella letteratura scolastica si tende a riconoscervi un segno del carattere atemporale e perenne della sua egemonia. La naturalizzazione di questo suo tratto indirizza la lettura e la comprensione dell'allievo, mentre dovrebbe invece fare posto a un insegnamento che evidenzi il processo di costruzione dei vari elementi culturali, fra i quali va inclusa la religione. Accanto all'ipertrofia del cristianesimo, vi è una caratterizzazione erronea $\mathrm{e}$ una minimizzazione del ruolo della religione romana. Non vi sono elementi testuali o iconografici sufficienti a coglierne i caratteri peculiari, come pure l'egemonia e la lunga durata. Un ambiente religioso plurale e dinamico viene così ridotto a una realtà religiosa fragile, lontana dai centri del potere e destinata alla sottomissione al cristianesimo. L'impero romano, in termini religiosi, viene visto sotto il segno di una somiglianza attualizzante, e non sotto quello della messa a fuoco storica: il contesto della fruizione dei libri didattici e la loro struttura argomentativa convergono verso una lettura che equipara i processi di cristianizzazione di Roma antica a quelli del Brasile attuale, rappresentando entrambi i contesti storici a partire da una visione di unità e di omogeneità religiosa, fondata sul cristianesimo.

\section{Conclusione}

Come ricordato in apertura, i libri di testo approvati dal PNLD e attualmente in uso nelle scuole brasiliane si basano su princìpi che dichiarano l'intento di difendere, tra l'altro, il pluralismo religioso e un insegnamento critico della Storia. L'analisi dettagliata delle discussioni che vi sono proposte riguardo allo sviluppo storico della religione nel mondo romano delinea però un quadro in larga misura differente. L'osservazione dei temi e dei silenzi ricorrenti nei testi e nelle immagini religiose in 
questi libri hanno confermato questa tendenza. In termini iconografici, la presentazione delle divinità romane e un'ipertrofia di immagini di Gesù. Nei testi si osserva un uso ridotto del termine "religione romana", mentre termini come "cristianesimo" e "religione cristiana" sono ampiamente ripetuti. Le analisi quantitative mostrano la stessa tendenza. L'uso di immagini non cristiane indica uno svuotamento religioso delle divinità romane e i testi che le accompagnano sostengono poco o nulla gli allievi nel riflettere sul contesto religioso romano. Nel caso delle immagini cristiane, vi è una sopravvalutazione dell'eroismo dei cristiani e della disfatta del nemico, l'impero romano. Il mondo romano viene così presentato come un ambiente dicotomico e non plurale, che si contrappone alla diversità sociale e religiosa dell'impero romano. Questo scenario è proposto da documenti, pitture e statue postclassiche, che implicano una serie di discorsi sui classici; anche i libri didattici discussi in questo lavoro ne mostrano la traccia. Da qui deriva la rilevanza della metodologia dei Reception Studies per la comprensione dei discorsi sui classici presenti nella letteratura didattica brasiliana ${ }^{65}$.

L'insoddisfacente quadro complessivo discusso in questo studio deriva dall'assenza pressoché completa di cenni al pluralismo religioso nel vasto dominio territoriale acquisito da Roma; dallo svuotamento delle divinità e dei culti romani e non romani; dalla marginalizzazione delle immagini religiose non cristiane, dall'ipertrofia di temi e immagini riferiti al cristianesimo, e dalla sua centralità politica e religiosa nell'impero romano. Molte delle immagini che contribuiscono a costituire questo scenario sono state elaborate con l'obiettivo di rafforzare l'identità cristiana, in Europa come in Brasile, a partire da un'idea di centralità dei classici nella storia occidentale ${ }^{66}$. Trascurando i contesti di produzione ed esibendo un quadro storico che ignora la religione romana, i libri di testo in uso nel Brasile contemporaneo alimentano ulteriormente questo discorso, quando dovrebbero invece accostarvisi criticamente, soprattutto a partire dalla presentazione del complesso paesaggio religioso romano e del lungo processo di consolidamento del cristianesimo a Roma. Ignorano così $\mathrm{i}$ molteplici livelli di interpretazione che hanno sorretto la comprensione moderna del mondo antico, trattando il passato come un oggetto di conoscenza immediata e conclusa, e non come l'esito dei contesti culturali entro i quali le sue letture antiche e moderne hanno preso forma.

\section{BIBLIOGRAFIA}

Alves, Oliveira 2016: A. Alves, L. Oliveira, Conexões com a história, São Paulo 2016.

AZEVEDO, SERIACOPI 2016: G. Azevedo, R. Seriacopi, História: Passado e presente. Dos primeiros humanos ao renascimento, São Paulo 2016.

BitTEnCOURT 2015: C. Bittencourt, O saber histórico na sala de aula, São Paulo 2015.

BOULOS JUNIOR 2016: A. Boulos Júnior, História Sociedade e Cidadania, São Paulo 2016.

BRAICK, MotA 2016: P. Braick, M. Mota, História das Cavernas ao Terceiro Milênio, São Paulo 2016. 
BUTLER 2016: S. Butler, Deep Classics: Rethinking Classical Reception, New York 2016.

CAmpos, Pinto, Claro 2016: F. Campos, J. Pinto, R. Claro, Oficina de História, São Paulo 2016.

CoTRIM 2016: G. Cotrim, História Global Ensino Médio, São Paulo 2016.

COSTA JUNIOR 2021: J. Costa Junior, Roma presente: Recepções clássicas em discursos políticos e educacionais no Brasil (2016-2020). Tesi di Dottorato, Universidade Federal do Estado do Rio de Janeiro, Rio de Janeiro 2021.

FoucAult 1971: M. Foucault, L'Ordre du discours, Paris 1971.

GoLDHILL 1995: S. Goldhill, Foucault's Virginity: Ancient Erotic Fiction and the History of Sexuality, Cambridge 1995.

GoLDHILL 2011: S. Goldhill, Victorian Culture and Classical Antiquity. Art, Opera, Fiction, and the Proclamation of Modernity, Princeton 2011.

GRANGEIRo 2016: C. Grangeiro, Cenas da História: Volume 1 Ensino Médio, São Paulo 2016.

GUARINELLO 2014: N. Guarinello, História Antiga, São Paulo 2014.

HARDWICK, STRAY 2008: L. Hardwick, C. Stray (eds.), A Companion to Classical Receptions, Oxford 2008.

HUNT, KHAN-Evans 2009: S. Hunt, A. Khan-Evans, «(R)evolution in Classics», in JACT INSET 2009. URL: https://www.academia.edu/22145265/R_evolution_in_Classics, ultimo accesso 27.03.21.

JoLY 2012: M. Joly, Introdução à análise da imagem, Campinas 2012.

MARques, BerutTi 2016: A. Marques, F. Berutti, Caminhos do Homem: das origens da humanidade à construção do mundo contemporâneo, São Paulo 2016.

MARTINDALE 2006: C. Martindale, «Thinking through Reception», in C. Martindale, R. Thomas (eds.), Classics and the Uses of Reception, London 2006, 1-13.

MARTINDALE 2007: C. Martindale, «Reception», in C. Kallendorf (ed.), A Companion to the Classical Tradition, Oxford 2007, 297-312.

MARTINDALE 2013: C. Martindale, «Reception - A New Humanism? Receptivity, Pedagogy, the Transhistorical», Classical Receptions Journal 5, 2013, 169-183.

MOEDE 2011: K. Moede, «Reliefs, Public and Private», in J. Rüpke (ed.), A Companion to the Roman Religion, Oxford 2011, 164-175.

MYLONOPOULOS 2010: J. Mylonopoulos, «Introduction - Divine Images versus Cult Images. An Endless Story about Theories, Methods, and Terminologies», in J. Mylonopoulos (ed.), Divine Images and Human Imaginations in Ancient Greece and Rome, Leiden-Boston 2010, 1-19.

OLIVEIRA 2013: N. Oliveira, História Antiga no Brasil: um perfil do ensino nas universidades brasileiras, Universidade Federal de Uberlândia 2013.

Pellegrini, DiAs, Grinberg 2016: M. Pellegrini, A. Dias, K. Grinberg, Contato História, São Paulo 2016. RIVES 2000: J. Rives, «Religion in the Roman World», in J. Huskinson (ed.), Experiencing Rome: Culture, Identity and Power in the Roman World, New York 2000, 245-277.

RÜPKE 2016: J. Rüpke, On Roman Religion: Lived Religion and the Individual in Ancient Rome, Ithaca 2016.

Santiago, Cerqueira, Pontes 2016: P. Santiago, C. Cerqueira, M. Pontes, Por dentro da História, São Paulo 2016. 
SQUIRE 2011: M. Squire, The Art of the Body: Antiquity and Its Legacy, London-New York 2011.

SQUIRE 2013: M. Squire, «Embodied Ambiguities on the Prima Porta Augustus», in Art History 36,

2013, 242-279.

VAINFAS, FARIA, FeRreIRA, SANTos 2016: R. Vainfas, S. Faria, J. Ferreira, G. Santos, História: Ensino

Médio, São Paulo 2016.

Vicentino, VinCEnTino 2016: C. Vicentino, B. Vicentino, Olhares da História: Brasil e Mundo, São Paulo

2016.

\section{NOTE}

1. La scelta dell'orizzonte temporale si spiega con tre considerazioni. In primo luogo, il PNLD-2018 è la serie più recente di libri di testo approvata dal governo federale brasiliano: le condizioni di accesso alla documentazione sono dunque ideali. Il Programa Nacional do Livro Didático è inoltre l'esito di un articolato processo di perfezionamento, che ha permesso l'elaborazione di materiali didattici di qualità più elevata e l'allargamento del quadro analitico. Infine, i libri di testo di Storia del PNLD-2018 sono il frutto di un lungo percorso di riflessione, che risale alla svolta democratica del 1985 e ha sostenuto il processo di rinascita della democrazia brasiliana dopo la dittatura militare (1964-1985). Nel 2018, però, l'elezione di Jair Bolsonaro alla Presidenza della Repubblica ha segnato un profondo mutamento nel quadro politico. Per la prima volta dal ritorno alla democrazia, i libri di testo circolano in un contesto dominato da un esponente di estrema destra, i cui valori sono diametralmente opposti a quelli che i manuali approvati dal PNLD si propongono di diffondere.

2. RÜPKE 2016, 42-43. In particolare, sui rapporti fra religione romana ed espansione imperiale, vd. RIVES 2000.

3. Vd. MYLONOPOULOS 2010, 18 e RÜPKE 2016, 62. Sul carattere divino delle immagini degli imperatori vd. in particolare SQUIRE 2011, 154-195.

4. Per una visione d'insieme sul PNLD e sulla platea di scuole e di studenti coinvolti dal programma vd. https://www.fnde.gov.br/index.php/programas/programas-do-livro/pnld/ dados-estatisticos, ultimo accesso 25.05.21.

5. Le scuole statali utilizzano necessariamente libri di testo approvati dal PNLD. Le scuole private e confessionali sono libere di scegliere i propri materiali pedagogici; il sigillo di approvazione del PNLD è però un forte incentivo anche per queste ultime.

6. Per un riepilogo sul contesto europeo vd. https://eurydice.indire.it/wp-content/uploads/ 2016/06/libri_testo_UE_2-1.pdf, ultimo accesso 15.07.21.

7. Le normative che codificano le idee esposte poco sopra includono la Costituzione Federale del 1988, la Lei de Diretrizes e Bases da Educação Nacional, i parametri dei curricula nazionali e le linee-guida curricolari nazionali.

8. Il concetto di un'educazione critica alla cittadinanza, dunque, non è soltanto un'aspirazione, ma l'obiettivo vero e proprio sul quale è orientata la legislazione in materia: il discorso nell'ambito educativo è sempre un luogo dell'esercizio del potere. In questi termini, come sostiene FouCAUlT 1971, spec. 10-12, la produzione del discorso è necessariamente controllata e manipolata, soprattutto in un ambito come la politica. Il rapporto fra strumenti normativi di carattere politico e giuridico e la produzione della letteratura didattica in Brasile si basa sugli stessi princìpi.

9. HUNT, KHAN-EVANS 2009, 5.

10. Cfr. Costa Junior 2021, 136. 
11. Mi pongo qui in continuità con l'idea di "ricezione" proposta da HARDWICK, STRAY 2008, 1: "By 'receptions' we mean the ways in which Greek and Roman material has been transmitted, translated, excerpted, interpreted, rewritten, re-imaged and represented. These are complex activities in which each reception 'event' is also part of wider processes".

12. Banca dati creata dalla Coordenação de Aperfeiçoamento de Pessoal de Nível Superior (CAPES) con l'intento di raccogliere le informazioni sulla produzione accademica nelle università brasiliane.

13. Su questo principio di lavoro vd. MARTINDALE 2007, GOLDHILL 2011 e BUTLER 2016.

14. I libri di testo analizzati qui sono rivolti agli studenti del primo anno dello Ensino Médio, nella fascia di età fra i 15 e i 16 anni.

15. La religione romana è costantemente definita "politeismo romano" o "paganesimo". Una discussione sulla pertinenza di questi termini esula dall'ambito della presente discussione.

16. Quando si farà riferimento a immagini non cristiane, si parlerà di "immagini romane". Quando necessario, si includerà un riferimento preciso: greche, celtiche, britanniche o egizie.

17. СотRIM 2016, 127.

18. Vicentino, VicENTino 2016, 160.

19. Pellegrini, Dias, Grinberg 2016, 162.

20. Santiago, Cerqueira, Pontes, 2016, 97.

21. AZEVEDO, SERIACOPI 2016, 132.

22. La Venere di Milo, nel libro Caminhos do Homem (MARQues, BERUTTI 2016, 8-9); la Venere di Fréjus, em História: Sociedade e Cidadania (Boulos JunioR, 2016, 157):

23. Si tratta di un esemplare in marmo prodotto intorno al 20 d.C. a partire da un originale in bronzo, elaborato fra il 27 e il 19 a.C. (SQUIRE, 2013, 135). La cronologia della statua marmorea o del suo modello bronzeo è il tema di un intenso dibattito fra gli specialisti, che esula dal tema di questo articolo. La statua di Cupido e Psiche è inclusa in Cenas de História (GRANGeIRo 2016, 86). L'Augusto di Prima Porta è in História das Cavernas ao Terceiro Milênio (BRAICK, MOTA 2016, 110), Olhares de História (VICENTINo, Vicentino 2016, 165), Caminhos do Homem (MARQUES, BERUTTI 2016, 97), Oficina de História (CAmpos, Pinto, Claro, 2016, 93), Cenas de História (GRAngeIro 2016, 95).

24. Pellegrini, Dias, Grinberg 2016, 161.

25. AZEVEDO, SERIACOPI 2016, 140.

26. СоTRIM 2016, 129.

27. AZEVEDO, SERIACOPI 2016, 149.

28. MARQUES, BEIRUTTI 2016, 8-9.

29. GRANGEIRO 2016, 111.

30. COTRIM 2016, 128.

31. AZEVEDO, SERIACOPI 2016, 155.

32. BOULOS JUNIOR 2016, 159

33. COTRIM 2016, 127.

34. BOULOS JUNIOR 2016, 158.

35. Pellegrini, Dias, Grinberg 2016, 163; Vainfas, Faria, Ferreira, Santos, 2016, 67.

36. BOULOS JUNIOR 2016, 158.

37. VicENTINO, ViCENTINO 2016, 170.

38. Alves, Oliveira 2016, 127.

39. Santiago, Cerqueira, Pontes, 2016, 108.

40. Alves, Oliveira 2016, 127.

41. La discussione della periodizzazione storica proposta nei libri di testo brasiliani esula dal tema di questo articolo. Basti qui dire che vi è una chiara traiettoria di "ascesa e declino" del potere politico di Roma nel Mediterraneo. Pur riconoscendo i margini di ambiguità e confusione 
insiti nel termine "caduta", lo si utilizzerà per riferirsi alla discussione proposta nei libri considerati in questo lavoro.

42. Riguardo ai rapporti fra rituali, immagini e religione romana, vd. M YLONOPOULOS 2010, 5-11, MOEDE 2011, 164; RÜPKE 2016, 61.

43. GOLDHILL 1995, 1-45.

44. Gli studi successivi sul tema potranno confermare se questa tendenza si riprodurrà anche nelle prossime serie di libri di Storia prodotti nel quadro del PNLD; tale sviluppo si spiegherebbe non solo con l'assenza di una formazione classica, ma anche con un'inerzia degli autori rispetto allo sviluppo degli studi su Roma antica.

45. Sull'analisi dell'immagine vd. Joly 2012. In particolare, sull'analisi delle immagini nei libri di testo, vd. BITTENCOURT 2015.

46. AZEVEDO, SERIACOPI 2016, 128.

47. Non è stato possibile riprodurre la pagina discussa qui. Disponibile a: https:// commons.wikimedia.org/wiki/File:Atenea_arcaizante.JPG Ultimo accesso: 12.07.21.

48. GRANGEIRO 2016, 86-87.

49. GRANGEIRO 2016,86 .

50. Berutti, Marques 2016, 97; Grangeiro 2016, 95; Braick, Mota 2016, 110; Campos, Pinto, Claro 2016, 93; VICENTINO, VICENTINO 2016, 165.

51. G RANGEIRO, 2016, 111. Disponibile a: https://commons.wikimedia.org/wiki/ File:Pintura_sobre_a_primeira_missa_no_Brasil.jpg. Ultimo accesso: 12.11.20. Non è stato possibile riprodurre la pagina discussa qui.

52. GRANGEIRO 2016, 112.

53. Sulla critica all'uso del termine "tradizione" in quanto lascito del passato al presente, vd. MARTINDALE 2006, 2007.

54. A dispetto del titolo, il libro di testo Historia Global non include una discussione del concetto di "globalità" nell'ambito degli studi storici. Il volume si configura piuttosto come una storia generale, ovvero come una discussione di grandi temi che non abbracciano direttamente la storia del Brasile.

55. História Global (CоTRIM 2016, 128). Non è stato possibile riprodurre la pagina discussa qui.

56. СоTRIM 2016, 128.

57. Ibid.

58. VICENTINO, VICENTINO 2016, 170.

59. Ibid.

60. Meriterebbe approfondire se i libri di testo discussi sin qui sottolineino l'importanza delle lotte religiose avvenute in altri periodi storici, e stabilire se si tratti di una precisa scelta editoriale degli autori: la discussione esula dai limiti di questo articolo.

61. Vd. https://g1.globo.com/politica/noticia/2020/01/13/50percent-dos-brasileiros-saocatolicos-31 percent-evangelicos-e-10percent-nao-tem-religiao-diz-datafolha.ghtml. Ultimo accesso: 11.04.21.

62. Vd. https://www1.folha.uol.com.br/ilustrissima/2019/09/relatos-apontam-proliferacao-deataques-as-religioes-afro-brasileiras.shtml e https://g1.globo.com/ba/bahia/noticia/ 2019/05/28/membros-de-terreiro-de-candomble-na-ba-denunciam-intolerancia-religiosa-aposato-de-grupo-casa-de-satanas.ghtml. Ultimo accesso: 15.04.21.

63. Vd. https://extra.globo.com/noticias/carnaval/tripe-de-cristo-oxala-da-mangueira-naodesfila-por-pressao-da-igreja-21011634.html._Ultimo accesso: 12.11.20.

64. Vd.: https://www.camara.leg.br/proposicoesWeb/fichadetramitacao?idProposicao= 491602 . Ultimo accesso: 12.11 .20 .

65. MARTINDALE 2006, 2013. 
66. L'identità cristiana alla quale ci si riferisce qui si associa alla tradizione in maggioranza cattolica del cristianesimo nella storia del Brasile, nonostante le correnti evangeliche si siano notevolmente diffuse a partire dagli anni Ottanta del secolo scorso.

\section{RIASSUNTI}

Lo studio di Roma antica, un tema ricorrente nella letteratura scolastica brasiliana sin dalle sue origini nel secolo XIX, si è orientato su due assi tematici principali: l'impero romano e l'ascesa del cristianesimo. Questo articolo intende discutere come i libri a carattere didattico in Brasile propongano una narrazione in cui il cristianesimo è presentato come la religione principale dell'impero romano, sminuendo per converso gli altri culti e i valori a essi associati. A partire dagli spunti metodologici proposti dai Reception Studies, si analizzerà la letteratura scolastica attualmente in uso, approvata dal governo brasiliano nel quadro del Programma Nazionale del Libro Didattico (PNLD-2018), entro il più ampio contesto delle tradizioni politico-religiose presenti e operanti nel Paese, e si sosterrà che questo materiale propone un paradigma didattico mirante a rappresentare come naturale e inevitabile l'attuale preponderanza del cristianesimo in Brasile.

The history of ancient Rome has been a set topic in Brazilian high schools since the nineteenth century, with a strong emphasis on two related themes: the Roman Empire and the rise of Christianity. This paper discusses how the schoolbooks currently in use in Brazil put forward a cohesive narrative in which Christianity appears as the only genuine religion in the Roman Empire, and, conversely, they downplay the historical significance of the other religions practiced in the Roman world. This paper, which builds on the methodological insights of Reception Studies, analyses the schoolbooks endorsed by the Brazilian government through the National Program of Schoolbooks (PNLD-2018) within the wider context of Brazilian political and religious traditions, and argues that the dominant teaching paradigm purposefully naturalises the Christian religious dominance in Brazil.

\section{INDICE}

Keywords : Roman Empire, Christianity, Brazil, schoolbooks, religious images, Classical reception

Parole chiave : Impero romano, cristianesimo, Brasile, letteratura didattica, immagini religiose, ricezione del classico

\section{AUTORE}

\section{JORWAN COSTA JUNIOR}

Universidade Federal do Estado do Rio de Janeiro,

Avenida Pasteur, 458, Urca,

Rio de Janeiro 22290-240, Brasil,

jorwangama(at)gmail.com 